\title{
ON THE FUNDAMENTAL GROUP SCHEME OF RATIONALLY CHAIN CONNECTED VARIETIES
}

\author{
MARCO ANTEI AND INDRANIL BISWAS
}

\begin{abstract}
Let $k$ be an algebraically closed field. Chambert-Loir proved that the étale fundamental group of a proper normal rationally chain connected variety over $k$ is finite. We prove that the fundamental group scheme of a proper normal rationally chain connected variety over $k$ is finite too. In particular, the fundamental group scheme of a Fano variety is finite.
\end{abstract}

\section{INTRODUCTION}

Let $X$ be a scheme over an algebraically closed field $k$ of characteristic $p$, with $p>0$. Fix a $k$-point $x$ of $X$. Nori introduced the notion of fundamental group scheme $\pi(X, x)$ in [12] and further developed it in [13]. Since then the fundamental group scheme is being studied and in the process has turned into an important tool in algebraic geometry of positive characteristic. In [13] Nori proves that $\pi(X, x)$ is trivial for proper rational normal varieties. More generally, $\pi(X, x)=0$ if $X$ is separably rationally connected [1]. Zhu proves that a general Fano (proper, smooth, connected with ample anticanonical bundle) hypersurfaces in projective spaces are separably rationally connected [16]. Therefore, the fundamental group schemes of general Fano hypersurfaces in a projective space are trivial.

In [3] Chambert-Loir proves that every proper rationally chain connected normal variety has finite étale fundamental group, and its order is coprime to $p$ (the characteristic of $k$ ) [4]. This result can also be obtained as a consequence of [8, Theorem 1.5] and [8, Theorem 1.6]. Shioda gave an example of a rationally connected variety over a field of characteristic $p \neq 5$ whose étale fundamental group is $\mathbb{Z} / 5 \mathbb{Z}$ [14]. Examples of rationally chain connected varieties whose local fundamental group scheme is not trivial are also known. For example, a supersingular Enriques surface $E$ over an algebraically closed field of characteristic 2 is unirational (see [5, Corollary 1.3.1]), hence it is rationally chain connected. It is known that there exists a nontrivial $\alpha_{2}$-torsor over $E$ (see [5, Chapter I, $\S 3])$.

We prove the following (see Theorem 3.10 and Remark 3.12):

Theorem 1.1. Let $k$ be an algebraically closed field and $X$ a proper normal, rationally chain connected $k$-scheme. Let $x \in X(k)$ be a point. Then the fundamental group scheme $\pi(X, x)$ is finite.

The strategy of the proof is similar to that in [3], adapted to the new setting.

2000 Mathematics Subject Classification. 14M22, 14H30. 


\section{Preliminaries}

We will write $\pi(X)$ instead of $\pi(X, x)$ to simplify the notation. However all the schemes for which we will compute the fundamental group scheme are meant to be pointed and all the morphisms between them take the marked point in the domain space to the marked point in the target space. The same convention will be applied to torsors: we assume they are pointed and morphisms between them take the marked point in the domain space to the marked point in the target space.

Let $k$ be an algebraically closed field of any characteristic. A proper variety $X$ over $k$ is said to be rationally chain connected if for every algebraically closed field $\Omega$ containing $k$, for any two points in $X(\Omega)$ there is a proper and connected curve passing through them such that its normalization is a disjoint union of projective lines. If this union consists of only one projective line we say that $X$ is rationally connected.

Let $X$ be a rationally chain connected variety over $k$. We recall [3, Lemma 1] and we sketch its proof:

Lemma 2.1. Let $k \subseteq \Omega$ be a field extension where $\Omega$ is algebraically closed. Let

$$
F_{\Omega}: \mathbb{P}_{\Omega}^{1} \longrightarrow X_{\Omega}
$$

be a rational curve of $X_{\Omega}$. Let $x_{0}:=F_{\Omega}(0)$ and $x_{\infty}:=F_{\Omega}(\infty)$ be points of $X_{\Omega}$ then let $V_{0}$ and $V_{\infty}$ be their Zariski closure in $X$. Then there exist a normal integral $k$-scheme $T$, a morphism

$$
F: \mathbb{P}_{T}^{1} \longrightarrow X
$$

such that the morphisms defined as

$$
F_{0}(t):=F(0, t): T \longrightarrow X \quad \text { and } \quad F_{\infty}(t):=F(\infty, t): T \longrightarrow X
$$

are dominant over $V_{0}$ and $V_{\infty}$ respectively.

Proof. There exists a finitely generated $k$-algebra $k \subseteq A$ contained in $\Omega$, and there is a morphism

$$
F_{A}: \mathbb{P}_{A}^{1} \longrightarrow X_{A}
$$

such that $F_{\Omega}=F_{A} \otimes_{A} \Omega$. We set $T:=\operatorname{Spec}(A)$ that we assume to be normal (otherwise we replace it with a finite extension). We now consider the morphism $F: \mathbb{P}_{T}^{1} \longrightarrow X$ obtained from $F_{A}$ after composing with the projection $X_{A} \longrightarrow X$. In [3, Lemma 1] it has been proved that $F_{0}$ and $F_{\infty}$ are dominant over $V_{0}$ and $V_{\infty}$; we briefly recall this last part for the convenience of the reader: We study $F_{0}$ (it will be the same for $F_{\infty}$ ). The image by $F_{0}$ of the generic point of $\operatorname{Spec}(A)$ is the generic point of $V_{0}$. Since $V_{0}$ is closed in $X$, the inverse image $F_{0}^{-1}\left(V_{0}\right)$ is closed in $\operatorname{Spec}(A)$ and dense. Thus $F_{0}^{-1}\left(V_{0}\right)$ coincides with $\operatorname{Spec}(A)$, and hence the image of $F_{0}$ is contained in $V_{0}$ and contains its generic point. Therefore it contains an open dense subset of $V_{0}$.

\section{THE MAIN THEOREM}

The following lemma is well-known. We include a short proof of it for the convenience of the reader. 
Lemma 3.1. Let $G$ be a finite $k$-group scheme and let $G^{\text {ét }}$ and $G^{\text {loc }}$ be respectively the maximal étale quotient and the maximal connected quotient. Then the natural morphism

$$
\alpha: G \longrightarrow G^{e ́ t} \times G^{\text {loc }}
$$

is faithfully flat.

Proof. The field being perfect the reduced subscheme $G_{\text {red }}$ is a subgroup scheme of

$$
N^{\text {loc }}:=\operatorname{kernel}\left(G \rightarrow G^{\text {loc }}\right),
$$

while the connected component $G^{0}$ of $G$ is $\operatorname{kernel}\left(G \rightarrow G^{\text {ét }}\right)$. If $\alpha$ is not faithfully flat we can factor it as in the following diagram:

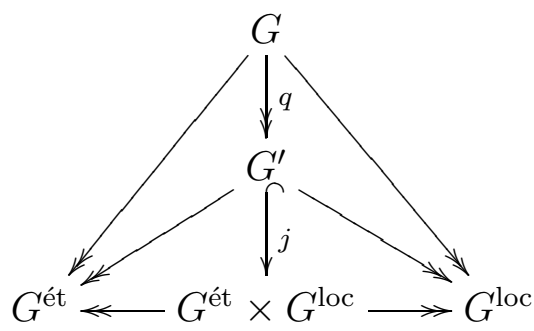

where $q: G \rightarrow G^{\prime}$ is faithfully flat and $j: G^{\prime} \hookrightarrow G^{\text {ét }} \times G^{\text {loc }}$ is a closed immersion. Clearly $G^{\text {ét }}$ and $G^{\text {loc }}$ are still the maximal étale quotient and the maximal connected quotient of $G^{\prime}$ respectively. So we can assume $\alpha$ is a closed immersion. Therefore, the lemma is equivalent to the assertion that $\alpha$ is an isomorphism.

From [15, $\S 6$, Ex. 9] it follows that $G^{\text {ét }}$ is isomorphic to a subgroup-scheme of $G$ which we identify with $G^{\text {ét }}$, so in particular $G^{\text {ét }} \leq G_{\text {red }} \leq N^{\text {loc }}$. Therefore, we have

$$
\left|G^{\text {ét }}\right|\left|G^{\text {loc }}\right|=\left|G^{\text {ét }}\right| \frac{|G|}{\left|N^{\text {loc }}\right|} \leq\left|G^{\text {ét }}\right| \frac{|G|}{\left|G^{\text {ét }}\right|}=|G|
$$

which implies that $\alpha$ is an isomorphism.

We recall that when $X$ is a reduced and connected scheme over a field $k$ then the fundamental group scheme can be defined. In this case a finite $G$-torsor $Y \longrightarrow X$ is called Nori-reduced if the canonical morphism $\pi(X) \longrightarrow G$ is faithfully flat.

Lemma 3.2. Let $X$ be a connected and reduced scheme over $k$. Let $G$ (respectively, $H$ ) be a finite local (respectively, finite étale) $k$-group scheme. Let

$$
Y \longrightarrow X
$$

and $T \longrightarrow X$ be a $G$-torsor and an $H$-torsor respectively. We assume that both $Y$ and $T$ are Nori-reduced. Then the $H \times G$-torsor

$$
T \times_{X} Y \longrightarrow X
$$

is also Nori-reduced.

Proof. If $\pi(X) \longrightarrow H \times G$ is not faithfully flat then there exists a triple $(M, Z, \iota)$, where

- $M \hookrightarrow H \times G$ is a subgroup-scheme,

- $Z \longrightarrow X$ is a $M$-torsor, and

- $\iota: Z \hookrightarrow T \times_{X} Y$ is a reduction of structure group-scheme, to $M$, of the $H \times G-$ torsor $T \times_{X} Y$. 
Let

$$
T^{\prime} \longrightarrow X \text { and } Y^{\prime} \longrightarrow X
$$

be the $M^{\text {ét }}$ and $M^{\text {loc }}$-torsors respectively, obtained from the $M$-torsor $Z \longrightarrow X$ using the projections of $M$ to $M^{\text {ét }}$ and $M^{\text {loc }}$ respectively (the notation is as in Lemma 3.1). We have a closed immersion

$$
Z \hookrightarrow T^{\prime} \times_{X} Y^{\prime}
$$

induced by the closed immersion $M \hookrightarrow M^{\text {ét }} \times M^{\text {loc }}$. The latter is an isomorphism by Lemma 3.1, so the same is true for $Z \hookrightarrow T^{\prime} \times_{X} Y^{\prime}$.

The projection $M \rightarrow H$ (respectively, $M \rightarrow G$ ) clearly factors through $M^{\text {ét }}$ (respectively, $\left.M^{\text {loc }}\right)$. Note that the projections

$$
M \longrightarrow H \text { and } M \longrightarrow G
$$

are faithfully flat morphisms because the two torsors $Y$ and $T$ are Nori-reduced. Consequently, the two homomorphisms $M^{\text {ét }} \longrightarrow H$ and $M^{\text {loc }} \longrightarrow G$ are isomorphisms. Now using Lemma 3.1 it follows that the inclusion $M \hookrightarrow H \times G$ is an isomorphism. Consequently, the $H \times G$-torsor $T \times_{X} Y \longrightarrow X$ is Nori-reduced.

The following result was proved in [7, Proposition 3.6] under the assumption that $X$ is proper.

Corollary 3.3. Let $X$ be a connected and reduced scheme over $k$. Let $G$ (respectively, $H$ ) be a finite local (respectively, finite étale) $k$-group scheme. Let $Y \longrightarrow X$ be a $G$-torsor and $T \longrightarrow X$ an $H$-torsor. We assume that both the torsors are Nori-reduced. Then the G-torsor

$$
T \times_{X} Y \longrightarrow T
$$

is also Nori-reduced. In particular, the morphism $\pi^{\mathrm{loc}}(T) \longrightarrow \pi^{\mathrm{loc}}(X)$ is faithfully flat.

Proof. Let us assume that there is a finite local $k$-group scheme $G_{1} \subset G$, and $G_{1}$-torsor $U \longrightarrow T$ and a reduction

$$
i: U \hookrightarrow T \times_{X} Y
$$

of structure group to $G_{1}$. Let $S$ be any $k$-scheme. For any $x \in X(S)$ we choose $u_{x} \in U(S)$ whose image in $X(S)$ is $x$. We set $\left(t_{x}, y_{x}\right):=i\left(u_{x}\right)$, then

$$
T(S) \times_{X(S)} Y(S)=\left\{\left(h t_{x}, g y_{x}\right), \quad \forall x \in X(S), \quad \forall g \in G(S), \quad \forall h \in H(S)\right\}
$$

so the image of $U(S)$ by $i_{S}$ can be identified with the subset

$$
\left\{\left(h t_{x}, g y_{x}\right), \forall x \in X, \forall g \in G_{1}, \quad \forall h \in H\right\} ;
$$

this gives $U$ the structure of an $H \times G_{1}$-torsor over $X$, contained in the $H \times G$-torsor $T \times_{X} Y$. This implies that $G_{1}=G$ by Lemma 3.2.

Corollary 3.4. Let $X$ be a connected reduced scheme over $k$ and $G$ a finite local $k$-group scheme. Let $T \longrightarrow X$ be a finite étale cover, and let $Y \longrightarrow X$ be a G-torsor. If $Y \longrightarrow X$ is Nori-reduced and $T$ is connected, then the $G$-torsor

$$
T \times_{X} Y \longrightarrow T
$$

is also Nori-reduced. In particular the homomorphism $\pi^{\mathrm{loc}}(T) \longrightarrow \pi^{\mathrm{loc}}(X)$ is faithfully flat. 
Proof. This follows from Corollary 3.3 and the fact that there exist a finite étale $k$-group scheme $H^{\prime}$ and an $H^{\prime}$-torsor $T^{\prime} \longrightarrow X$ that dominates $T \longrightarrow X$.

Remark 3.5. In [13], Nori proved that if $i: U \longrightarrow Y$ is an open immersion between connected and reduced schemes with $Y$ normal, then the morphism $\pi(U) \longrightarrow \pi(Y)$ induced by $i$ is faithfully flat (see $\S$ II, Proposition 6 and its corollaries). Consequently, the homomorphism $\pi^{\mathrm{loc}}(U) \longrightarrow \pi^{\mathrm{loc}}(Y)$ induced by $i$ is also faithfully flat.

Notation 3.6. Let $k$ be a field and $u: M \longrightarrow G$ a $k$-group scheme homomorphism. We say that $u$ is of finite index if the following property is satisfied: for any $k$-group scheme $Q$ and any faithfully flat morphism of $k$-group schemes $G \longrightarrow Q$, if the group scheme image of $M \rightarrow Q$ is finite then $Q$ is also finite.

Lemma 3.7. Let $f: X \longrightarrow Y$ be a finite purely inseparable morphism between normal integral schemes. Then the homomorphism

$$
\pi(X) \longrightarrow \pi(Y)
$$

is of finite index, while $\pi^{\mathrm{e} t}(X) \longrightarrow \pi^{\text {ét }}(Y)$ is faithfully flat. So in particular $\pi^{\text {loc }}(X) \longrightarrow$ $\pi^{\operatorname{loc}}(Y)$ is of finite index.

Proof. We assume that $\operatorname{char}(k)=p>0$. We observe that under the above assumptions the morphism $f$ is surjective (see [10, Ex. 5.3.9]). Let us first consider the case where $Y=X$ with $f:=F_{Y}$ being the absolute Frobenius morphism of $Y$. Let $T \longrightarrow Y$ be the universal $\pi(Y)$-torsor of $Y$ (it is a scheme, as all the transition morphisms are affine), where $\pi(Y)$ is the fundamental group scheme of Nori. We set

$$
T^{(p)}:=T \times_{Y} Y
$$

via the Frobenius $F_{Y}$ of $Y$. As usual, $F_{T / Y}: T \longrightarrow T^{(p)}$ is the relative Frobenius. The relative Frobenius commutes with base change, so if we pull back over $x: \operatorname{Spec}(k) \longrightarrow Y$ (a fixed closed point) what we obtain is the relative Frobenius

$$
F_{\pi(Y) / \operatorname{Spec}(k)}: \pi(Y) \longrightarrow \pi(Y)^{(p)} \simeq \pi(Y)
$$

where the last isomorphism clearly follows from the fact that $k$ is algebraically closed, whence perfect; thus, in particular,

$$
F_{T / Y}: T \longrightarrow T^{(p)}
$$

is the natural morphism from the universal torsor to the pro-finite torsor obtained after pulling back. The same holds for any torsor: so let $P$ be a $Q$-torsor, where $\pi(Y) \longrightarrow Q$ is a faithfully flat $k$-group scheme homomorphism; then we have the relative Frobenius

$$
F_{Q / \operatorname{Spec}(k)}: Q \longrightarrow Q^{(p)} \simeq Q
$$

which factors as $Q \longrightarrow F \longrightarrow Q$ (where $Q \longrightarrow F$ is faithfully flat and $F \longrightarrow Q$ is a closed immersion). Since the kernel is finite, if we assume $F$ to be finite then $Q$ is also finite thus $F_{\pi(Y) / \operatorname{Spec}(k)}$ is of finite index.

Now $F_{\pi(Y) / \operatorname{Spec}(k)}$ is a finite endomorphism and this is sufficient to conclude that it is of finite index. As étale torsors are not modified by the Frobenius, it follows that $F_{\pi^{\text {et }}(Y) / \operatorname{Spec}(k)}$ is an isomorphism. What has been proved for $f=F_{Y}$ still holds, of course, for $f=F_{Y}^{m}$, the Frobenius iterated $m$ times. So now we consider the general case where 
$f: X \longrightarrow Y$ is the given purely inseparable morphism. Then there exist a positive integer $m$ and a morphism $h: Y \longrightarrow X$ such that

$$
f \circ h=F_{Y}^{m}: Y \longrightarrow Y
$$

(the absolute Frobenius morphism iterated $m$ times). We consider the pullback

$$
T_{X}:=T \times_{Y} X
$$

and the universal $\pi(X)$-torsor $P \longrightarrow X$ on $X$. There are natural morphisms $P \longrightarrow T_{X}$ and

$$
u: \pi(X) \longrightarrow \pi(Y) .
$$

Pulling back further to $h: Y \longrightarrow X$, the following factorization is obtained:

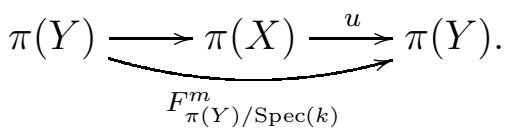

The previous discussion yield the following:

- if we assume that $\pi(Y)$ and $\pi(X)$ are both étale, this implies that

$$
u: \pi(X) \longrightarrow \pi(Y)
$$

is faithfully flat;

- otherwise we can only conclude that $u$ is of finite index, which is all that we can expect.

This is enough to conclude the proof.

Lemma 3.8. Let $f: X \longrightarrow Y$ be a dominant morphism between normal integral schemes. Then the homomorphisms

$$
\varphi^{\mathrm{loc}}: \pi^{\mathrm{loc}}(X) \longrightarrow \pi^{\mathrm{loc}}(Y) \text { and } \varphi^{\mathrm{ét}}: \pi^{\mathrm{ét}}(X) \longrightarrow \pi^{\mathrm{e} t}(Y)
$$

induced by $f$ are of finite index.

Proof. This is inspired by [3, Lemme 2] (see also [6, Lemme 4.4.17] for the zero characteristic case).

Let $t$ be a closed point of the generic fiber of $f$, and let $T$ denote its Zariski closure in $X$. The morphism $f$ induces a generically finite morphism $f_{\mid T}: T \longrightarrow Y$ : indeed its generic fiber has relative dimension zero and it this thus a finite number of points; therefore, there exists an open dense subscheme $U \subseteq Y$ such that

$$
f^{\prime}: V \longrightarrow U
$$

where $V:=T \times_{Y} U$, is a finite morphism. Hence there exist a scheme $W$ and two finite morphisms

$$
e: V \longrightarrow W \text { and } i: W \longrightarrow V
$$

such that $i \circ e=f^{\prime}$, where $i$ is purely inseparable and $e$ is generically étale. This implies that there exists an open dense subscheme $W^{\prime} \subseteq W$ such that

$$
e^{\prime}: V^{\prime} \longrightarrow W^{\prime} \text {, }
$$

where $V^{\prime}:=V \times_{W} W^{\prime}$, is a finite étale cover. In what follows we study the morphism

$$
\varphi^{\mathrm{loc}}: \pi^{\mathrm{loc}}(X) \longrightarrow \pi^{\mathrm{loc}}(Y)
$$


the (similar) details for $\varphi^{\text {ét }}$ are left to the reader. By Corollary 3.4 the morphism $\pi^{\mathrm{loc}}\left(V^{\prime}\right) \longrightarrow \pi^{\mathrm{loc}}\left(W^{\prime}\right)$ induced by $e^{\prime}$ is faithfully flat while the morphism

$$
\pi^{\mathrm{loc}}(W) \longrightarrow \pi^{\mathrm{loc}}(U)
$$

induced by $i$ is of finite index by Lemma 3.7 and clearly $\pi^{\text {loc }}\left(W^{\prime}\right) \longrightarrow \pi^{\text {loc }}(W)$ is faithfully flat (see Remark 3.5) so the composition

$$
u: \pi^{\mathrm{loc}}\left(V^{\prime}\right) \longrightarrow \pi^{\mathrm{loc}}(U)
$$

is of finite index. Now we have the diagram of homomorphisms of local fundamental group schemes

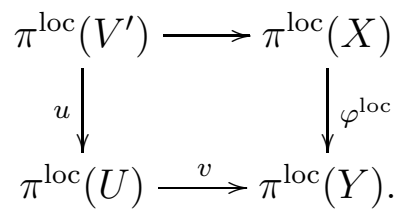

Now $u$ is of finite index and the homomorphism $v$ is faithfully flat (see, again, Remark 3.5). Hence $\varphi^{\text {loc }}$ is finite index.

In [11] Mehta and Subramanian proved that

$$
\pi(X \times Y)=\pi(X) \times \pi(Y)
$$

for two connected, proper and reduced schemes $X$ and $Y$. If one of the two schemes ( $X$ or $Y$ ) is not proper anymore then the previous formula may not hold. However a weaker result will be sufficient for our purposes; the following proposition can be found in [13, Chapter II, Proposition 9, here we suggest a different approach :

Proposition 3.9. Let $Y$ be an integral scheme over $k$, then the homomorphism

$$
\varphi: \pi\left(\mathbb{P}^{1} \times Y\right) \longrightarrow \pi(Y)
$$

induced by the projection $p_{2}: \mathbb{P}^{1} \times Y \longrightarrow Y$ is an isomorphism.

Proof. It is clear that $\varphi$ is faithfully flat as $p_{2}$ has a section, so we only need to prove that given a finite $k$-group scheme $G$ and a $G$-torsor $T \longrightarrow \mathbb{P}^{1} \times Y$, there exists a $G$-torsor

$$
T^{\prime} \longrightarrow U
$$

whose pullback to $\mathbb{P}^{1} \times Y$ is the given one. First we briefly recall that the fundamental group scheme of $Y$ at a $k$-point $y$ is the automorphism group scheme of the fiber functor $y^{*}$ on the category of essentially finite vector bundles, as described in [12]. Let $\operatorname{Rep}_{k}(G)$ denote the category of $k$-linear finite dimensional representations of $G$. Then associated to our $G$-torsor $T \longrightarrow \mathbb{P}^{1} \times Y$ there is a fiber functor

$$
F_{T}: \operatorname{Rep}_{k}(G) \longrightarrow \mathcal{Q} \operatorname{coh}\left(\mathbb{P}^{1} \times Y\right)
$$

by a fundamental result in Tannakian theory (recalled for instance in [12, Proposition $(2.9)])$. From this we will construct a functor

$$
F: \operatorname{Rep}_{k}(G) \longrightarrow \mathcal{Q} \operatorname{coh}(Y) \text {. }
$$

For any $G$-module $V$, set

$$
F(V)=\left(p_{2}\right)_{*}\left(F_{T}(V)\right)
$$


We first observe that $F(V)$ is a vector bundle: when restricted to $\mathbb{P}^{1}$, clearly $F_{T}(V)$ is an essentially finite vector bundle over the projective line, thus trivial, whence $H^{1}\left(\mathbb{P}^{1}, F_{T}(V)\right)=$ 0 , and the evaluation homomorphism

$$
\left(p_{2}\right)^{*}\left(p_{2}\right)_{*}\left(F_{T}(V)\right) \longrightarrow\left(F_{T}(V)\right)
$$

is an isomorphism. Moreover $F$ is compatible with the operations of taking tensor products, direct sums and duals. Hence $F$ is a fiber functor and we can associate to it a $G$-torsor $T^{\prime} \longrightarrow U$ which is the desired one since its pullback to $\mathbb{P}^{1} \times Y$ is isomorphic to $T \longrightarrow \mathbb{P}^{1} \times Y$.

We now recall that for any $0 \leq \nu \leq \operatorname{dim}(X)$, there is a point in $X(\Omega)$, where $\Omega$ in the algebraic closure of the function field of $X$, whose Zariski closure in $X$ is of dimension $\nu$.

Theorem 3.10. Let $k$ be an algebraically closed field and $X$ a normal, rationally chain connected $k$-scheme. Then $\pi^{\mathrm{loc}}(X)$ is finite.

Proof. Since $X$ is rationally chain connected, there exists a chain of rational curves connecting a rational point $x_{0} \in X(k)$ to a generic point $x_{m} \in X(\Omega)$, where $\Omega$ is the algebraic closure of the function field of $X$. According to Lemma 2.1 there exists a sequence of integral subvarieties $V_{0}, \cdots, V_{m}$ of $X$ where $V_{0}=x_{0}$ and $V_{m}=X$ and for every integer $i \in\{0, \cdots, m-1\}$ a family of rational curves

$$
F^{i}: \mathbb{P}_{k}^{1} \times T_{i} \longrightarrow X
$$

with $T_{i}$ normal and projective, such that the morphisms

$$
F_{0}^{i}: T_{i} \longrightarrow X \quad \text { and } \quad F_{\infty}^{i}: T_{i} \longrightarrow X
$$

defined by $F_{0}^{i}(t):=F^{i}(0, t)$ and $F_{\infty}^{i}(t):=F^{i}(\infty, t)$, are dominant on $V_{i}$ and $V_{i+1}$ respectively. If $V_{i}$ is not normal then we can consider an open normal subscheme $V_{i}^{\prime} \subset V_{i}$ and the pullback

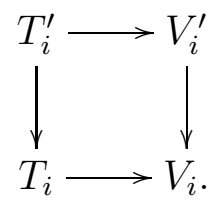

In a similar way, if $V_{i+1}$ is not normal then we can consider an open normal subscheme $V_{i+1}^{\prime} \subset V_{i+1}$ and its pullback, as before, that we will call $T_{i}^{\prime \prime}$. This will not affect $V_{0}$ and 
$V_{m}$ of course. So this induces the following commutative diagram on local group schemes:

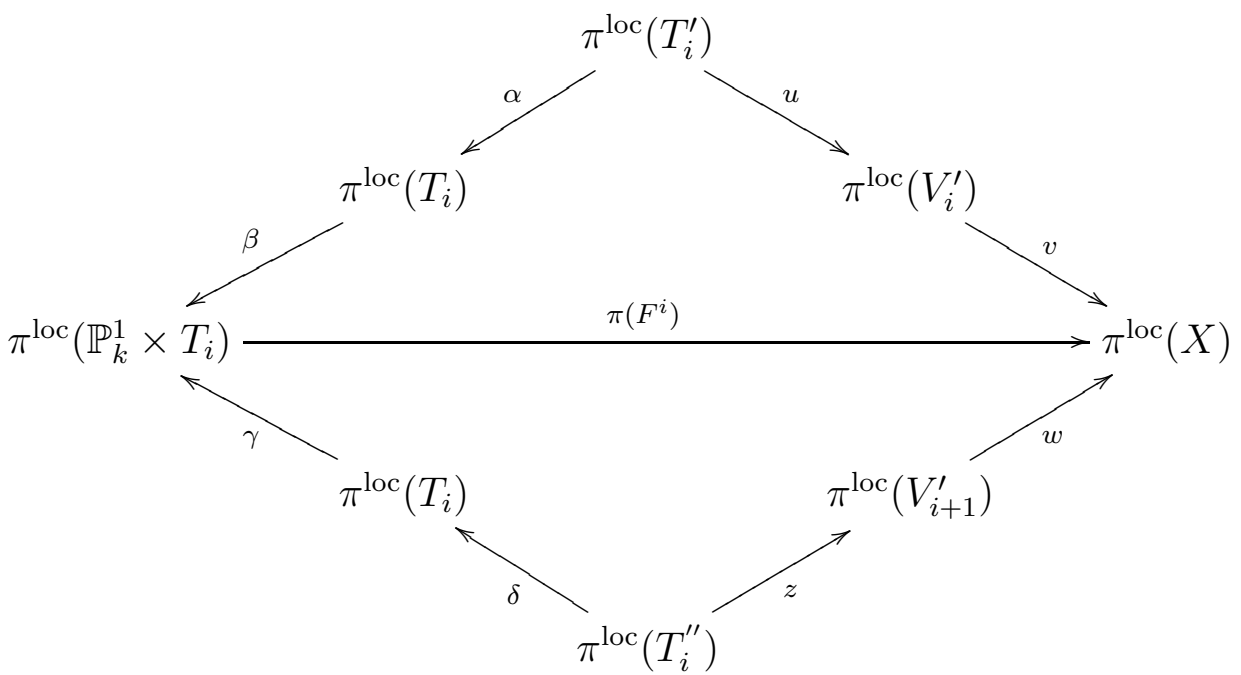

We avoid to put the index $i$ on the morphisms not to make notation too heavy. We know that $\pi^{\operatorname{loc}}\left(V_{0}\right)=0$, both $u$ and $z$ are of finite index by Lemma 3.8, both $\alpha$ and $\delta$ are faithfully flat by Remark 3.5 and both $\beta$ and $\gamma$ are isomorphisms by Proposition [3.9. So at each step we prove that the image of $\pi^{\text {loc }}\left(V_{i+1}^{\prime}\right)$ in $\pi^{\text {loc }}(X)$ is finite. The last step will finally prove that $\pi^{\text {loc }}(X)$ is finite.

Lemma 3.11. Let $X$ be a rationally chain connected variety and let $f: Y \longrightarrow X$ be an étale Galois cover. Then $Y$ is rationally chain connected.

Proof. Fix a point $y$ of $Y$. Let $U_{y}$ be the subset of $Y$ that is rationally chain connected to the point $y$. Let $\Omega$ be any algebraically closed field containing $k$, then any morphism $g: \mathbb{P}_{\Omega}^{1} \longrightarrow X_{\Omega}$ lifts to a morphism $g^{\prime}: \mathbb{P}_{\Omega}^{1} \longrightarrow Y_{\Omega}$ using the homotopy lifting property because $\mathbb{P}_{\Omega}^{1}$ is simply connected. Since $X$ is also rationally chain connected, these two together imply that $U_{y}$ is both open and closed. Hence $U_{y}=Y$, and $Y$ is rationally chain connected.

Remark 3.12. Let notations be as in Theorem[3.10, then from [3, Théorème] and Lemma 3.11 we also obtain that $\pi(X)$ is finite. If $\operatorname{char}(k)=p>0$, and $X$ is moreover smooth and proper, then $\left|\pi(X)^{\text {ét }}\right|$ is coprime to $p$, as proved in [4]. In particular all this holds when $X$ is a Fano variety since in this case it is rationally chain connected (cf. [2] and [9]). Furthermore when $X$ is a general hypersurface of a projective space then then it is separably rationally connected, and by [1] this implies that $\pi(X)=0$.

\section{ACKNOWLEDGMENTS}

We thank Antoine Chambert-Loir for a useful communication. We thank the two referees for comments that helped us in improving the paper. The first-named author would like to thank T.I.F.R. for its hospitality and Cinzia Casagrande for useful discussion. The second-named author thanks Université Lille 1 and Niels Borne for hospitality. He also acknowledges the support of a J. C. Bose Fellowship. 


\section{REFERENCES}

[1] I. Biswas, On the fundamental group-scheme, Bull. Sci. Math. 133 (2009), 477-483.

[2] F. Campana, Connexité rationnelle des variétés de Fano, Ann. Sci. École Norm. Sup. 25 (1992), $539-545$.

[3] A. Chambert-Loir, À propos du groupe fondamental des variétés rationnellement connexes, $C$. $R$. Acad. Sci. Paris, Ser. I (2003), arXiv:math/0303051v1 [math.AG].

[4] A. Chambert-Loir, Points rationnels et groupes fondamentaux: applications de la cohomologie p-adique (d'après P. Berthelot, T. Ekedahl, H. Esnault, etc.), Astérisque No. 294 (2004), 125-146.

[5] F. R. Cossec and I. V. Dolgachev, Enriques surfaces. I. Progress in Mathematics, 76. Birkhäser Boston, Inc., Boston, MA, 1989.

[6] P. Deligne, Théorie de Hodge : II, Inst. Hautes Études Sci. Publ. Math. 40 (1971), 5-57.

[7] H. Esnault, P. H. Hai And X. Sun, On Nori's fundamental group scheme. Geometry and dynamics of groups and spaces, 377-398, Progr. Math., 265, Birkhäuser, Basel, 2008.

[8] J. Kollár, Shafarevich maps and plurigenera of algebraic varieties Invent. Math. 113 (1993), 177215.

[9] J. Kollár, Y. MiYaOka and S. Mori, Rational connectedness and boundedness of Fano manifolds, Jour. Diff. Geom. 36 (1992), 765-779.

[10] Q. Liu, Algebraic geometry and arithmetic curves. Oxford Graduate Texts in Mathematics 6 Oxford University Press, 2006.

[11] V. B Mehta and S. Subramanian, On the fundamental group scheme, Invent. Math. 148 (2002), $143-150$.

[12] M. V. Nori, On the representations of the fundamental group, Compos. Math. 33 (1976), 29-42.

[13] M. V. Nori, The fundamental group-scheme, Proc. Ind. Acad. Sci. (Math. Sci.) 91 (1982), 73-122.

[14] T. Shioda, An example of unirational surfaces in characteristic p, Math. Ann. 211 (1974), 233-236.

[15] W. C. Waterhouse, Introduction to affine group schemes, Graduate Texts in Mathematics, 66. Springer-Verlag, New York-Berlin, 1979.

[16] Y. ZHU, Fano hypersurfaces in positive characteristic, arXiv:1111.2964v1 [math.AG].

Laboratoire J.A.Dieudonné, UMR CNRS-UnS No 7351 Université de Nice Sophia-Antipolis, Parc Valrose, 06108 NiCE Cedex 2, France

E-mail address: Marco.ANTEI@unice.fr

School of Mathematics, Tata Institute of Fundamental Research, Homi Bhabha Road, BOMBAY 400005, INDIA

E-mail address: indranil@math.tifr.res.in 